\title{
Hemophagocytic Syndrome Associated with Cytomegalovirus Infection in a Severely Immunocompromised AIDS Patient: Case Report
}

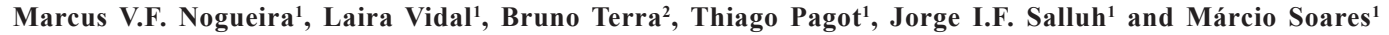 \\ ${ }^{1}$ Intensive Care Unit, National Institute of Cancer; ${ }^{2}$ Department of Hematology and Department of Clinical Research, National Institute of \\ Cancer; Rio de Janeiro, RJ, Brazil
}

\begin{abstract}
Hemophagocytic syndrome is a clinical condition characterized by the infiltration of the bone marrow and reticuloendothelial system by macrophages and activated histiocytes, leading to uncontrolled phagocytosis of platelets, erythrocytes, lymphocytes and precursor cells. It is a severe inflammatory and aggressive condition, characterized by high fever, hepatosplenomegaly, lymphadenopathy and cytopenia, and it may lead to organ dysfunction. This syndrome is classified as familial or acquired; the latter is more frequent and is associated with diverse conditions, such as infections, malignancies and rheumatic diseases. We report a case of HLH associated with cytomegalovirus infection in a patient with acquired-immunodeficiency syndrome and Burkitt's lymphoma.
\end{abstract}

Key-Words: Hemophagocytic syndrome, histiocytes, immunosuppression, sepsis, organ dysfunction.

Hemophagocytic syndrome or hemophagocytic lymphohistiocytosis (HLH) is a clinical condition characterized by infiltration of the bone marrow and reticuloendothelial system by macrophages and activated histiocytes, leading to uncontrolled phagocytosis of platelets, erythrocytes, lymphocytes and precursor cells. HLH is traditionally considered a rare event, but recent studies have suggested that this condition is underdiagnosed. HLH is a severe inflammatory and aggressive condition, characterized by high fever, hepatosplenomegaly, lymphadenopathy and cytopenia. Central nervous system involvement, cutaneous manifestations, severe coagulation disturbances and multiple organ dysfunctions occur less frequently. Laboratory findings of high levels of ferritin, hypertriglyceridemia and hypofibrinogenemia can also be present, supporting the diagnosis.

This syndrome is classified as familial or acquired, the latter being more frequent. The familial form is related to genetic immune defects, while the acquired form is associated with several etiologies, including viral [Epstein-Barr Virus (EBV), cytomegalovirus (CMV), and human immunodeficiency virus (HIV)], bacterial, fungal and protozoa infections, malignancies (lymphomas), and other conditions, such as rheumatic diseases.

We report a case of HLH associated with cytomegalovirus infection in a patient with acquired-immunodeficiency syndrome (AIDS) and Burkitt's lymphoma.

Data were obtained from patient's charts and medical records. Written informed consent was obtained from the patients' relatives. The institutional review board approved the study.

Received on 16 July 2008; revised 28 January 2009.

Address for correspondence: Dr. Márcio Soares, MD, PhD. Instituto Nacional do Câncer - INCA, Centro de Tratamento Intensivo - $10^{\circ}$ Andar. Pça. Cruz Vermelha, 23 - Rio de Janeiro - RJ - Brazil. Zip code: 20230-130. Phone: +55 21 25066120; Fax: +55 21 22948620. Email: marciosoaresms@yahoo.com.br, marciosoaresms@gmail.com.

\section{Case Report}

A 34 year-old man with AIDS who was on antiretroviral therapy (stavudine, lamivudine, atazanavir and ritonavir) was admitted to the hospital with a cervical mass. An excisional biopsy yielded a diagnosis of Burkitt's lymphoma. The CD4 T-lymphocyte count was 386 cells $/ \mathrm{mm}^{3}$, and the viral load was undetectable. One week later, chemotherapy was initiated, with a significant reduction in the size of the cervical mass, and the patient was discharged. Three days later, he was readmitted to the hospital with febrile neutropenia (39 neutrophils $/ \mathrm{mm} 3$ ), thrombocytopenia $(39,000 / \mathrm{mm} 3)$ and mucositis. Despite the administration of broad-spectrum antibiotics, the patient developed acute respiratory failure with a need for mechanical ventilation; he was transferred to the intensive care unit (ICU). Pneumocystitis jirovecci pneumonia was diagnosed and treated with trimethropim-sulfamethoxazole, prednisone and granulocyte colony-stimulating factor (GCS-F) was started. The patient's clinical condition improved and his blood cell count normalized. During the ICU stay, the patient developed ventilator-associated pneumonia. Despite appropriate antimicrobial therapy, he evolved with persistent fever and pancytopenia (leukocyte count: $800 / \mathrm{mm}^{3}$; platelet count: $14,000 / \mathrm{mm}^{3}$; and hemoglobin level: $6.5 \mathrm{~g} / \mathrm{dL}$ ). GCS-F was administered again, with no response. Several diagnostic procedures to determine the etiology of the pancytopenia were performed. Cytomegalovirus antigenemia was positive, and the bone marrow aspirate revealed hypoplasia, with histiocyte infiltration, as well as phagocytosis of blood cells, consistent with HLH (Figure 1). Based on these findings, therapy was initiated with ganciclovir, resulting in resolution of fever and recovery of the blood cell count. Ten days later, the patient was discharged from the ICU. In the hospital room, cell blood counts returned to normal, CMV antigenemia was negative and the patient was discharged from the hospital.

\section{Discussion}

The hemophagocytic syndrome was first described in 1979 in immunosuppressed patients with viral infections [1]. EBV is the most common etiology, while CMV is associated with $30 \%$ to $40 \%$ of all virus-associated HLH cases [2]. This 
Figure 1. Histiocytes with phagocytosis of erythrocytes and platelets.

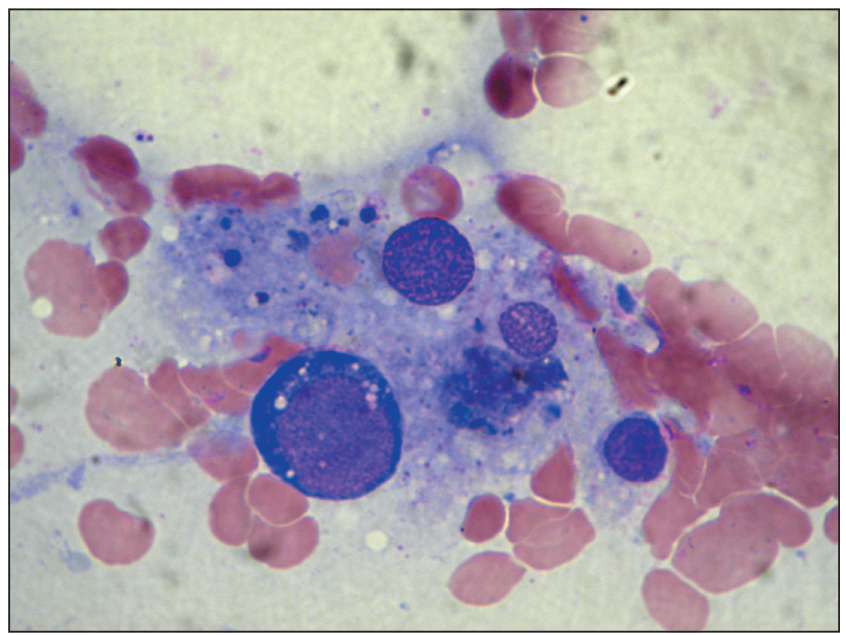

syndrome is a result of damage caused by cytotoxic activity of natural killer (NK) cells and T cells; thus the ineffective immune system is constantly stimulated to generate high levels of cytokines, such as tumor necrosis factor $\alpha$ (TNF- $\alpha)$ and interferon $\gamma$ (IFN- $\gamma$ ), which stimulate the defense cells. High levels of cytokines are responsible for the clinical picture [2,3]. HLH is an uncommon and frequently undiagnosed event, recently described in critically-ill patients [2-4]; it remains a diagnostic challenge, as the clinical presentation of this condition mimics sepsis, a frequent syndrome in ICU patients [5]. Laboratorial tests for diagnosis, such as serum triglyceride, ferritin and fibrinogen levels, are frequently not performed in the ICU, and they lack specificity, as they may be significantly altered as a result of critical illness. Moreover, blood transfusions may mask pancytopenia. Our patient had at least four conditions associated with the development of HLH (HIV infection, Burkitt's lymphoma, bacterial sepsis and CMV infection). The association of HLH and HIV has been described; however, it occurs less frequently than previously expected [6]. HIV-positive patients with lymphoma are at high risk for HLH [1,3]. The association between bacterial infection and HLH is poorly documented, and most case reports indicate that it is due to intracellular bacteria (e.g. Mycobacteria, Legionella sp.) [1]. Finally, although the other factors could cause HLH, we believe that CMV was the main etiologic agent involved. The prompt response to ganciclovir allowed clinical and hematological improvement and ICU discharge. This case emphasizes the importance of bone marrow examination and extensive investigation for opportunistic infections in immunocompromised patients presenting with febrile pancytopenia. In most cases, treatment of the underlying condition promotes complete remission of the clinical picture.

\section{Conclusions}

We report a case of HLH associated with cytomegalovirus infection in a patient with AIDS and Burkitt's lymphoma. Although HLH is an uncommon event described in critical patients, it is possibly underdiagnosed. It has been associated with a series of infectious agents, as well as genetic, autoimmune and neoplastic diseases. Awareness concerning its clinical presentation and a high level of clinical suspicion are essential, especially in immunosuppressed patients with fever, hepatosplenomegaly, and pancytopenia that do not respond to GCS-F and antibiotics. Treatment of the underlying condition often leads to clinical improvement.

\section{References}

1. Risdall R.J., McKenna R.W., Nesbit M.E., et al. Virus-associated hemophagocytic syndrome: a benign histiocytic proliferation distinct from malignant histiocytosis. Cancer 1979;44:9931002.

2. Créput C., Galicier L., Buyse S., Azoulay E. Understanding organ dysfunction in hemophagocytic lymphohistiocytosis. Intensive Care Med 2008;34:1177-87.

3. Janka G.E. Hemophagocytic syndromes. Blood Rev 2007;21:24553.

4. Fisman D.N. Hemophagocytic syndromes and infection. Emerg Infect Dis 2000;6:601-8.

5. Soares M., Salluh J.I. Validation of the SAPS 3 admission prognostic model in patients with cancer in need of intensive care. Intensive Care Med 2006;32:1839-44.

6. Pastore R.D., Godwin J.H. HIV/AIDS case histories: diagnostic problems. Hemophagocytic syndrome. AIDS Patient Care STDS 1999; $13: 677-8$. 